\title{
Effect of the Width of the NRD Waveguide on the S Parameter of a Pass Band Filter
}

\author{
Harizi Hanen ${ }^{1}$, Lattrach Lassaad ${ }^{1}$, Gharssallah Ali $^{1}$, Garbi Abdelhafedh ${ }^{2}$ \\ ${ }^{1}$ Unit of Research Circuits and Electronics Systems High Frequency, Faculty of Science, University El Manar, Tunis, Tunisia; \\ ${ }^{2}$ Laboratory of Research Circuits and Electronics Systems High Frequency, Faculty of Science, University El Manar, Tunis, Tunisia. \\ Email: hanenharizi@gmail.com
}

Received July $9^{\text {th }}$, 2012; revised August $18^{\text {th }}$, 2012; accepted September $3^{\text {rd }}$, 2012

\begin{abstract}
In this paper, we present a design of a non-radiative dielectric waveguide band pass filter based on hybrid architecture of micro-strip line and non-radiative dielectric waveguide. The simulation with high frequency structure simulator (HFSS) three dimensional analyses are presented, also the influence of the feeding transitions for circuit design is studied. The aim of this work was to study the influence of the width of the NRD waveguide to scattering parameters.
\end{abstract}

Keywords: Non Radiative Dielectric (NRD)

\section{Introduction}

The technology demand to realize high efficiency circuit, but productions cost extremely high. Designed for millimetre wave, active circuits use planar technology and passive circuits employ waveguide technologies to integer those two technologies require highly accurate assembly thus increasing production cost and time. To decrease it, the non radiative dielectric waveguide technology should be used.

A non-radiative dielectric waveguide is currently interesting filter types due to their high $\mathrm{Q}$ at low cost technology for fabrication and compatibility with other technology.

Hence their electromagnetic models are still under research and therefore not easy accessible. This work aims to provide circuit designers with accurate, easy determinable for a pass band filter.

Proposed by Yoneyama and Nishida in 1981 [1], nonradiative dielectric (NRD) waveguide circuits is nowadays a well-known technology for various millimeterwave applications [2,3]. Subsequently, Bacha has proposed the model of a hybrid integration of NRDwaveguide and micro-strip line [4,5]. Indeed, basic features and applications of the NRD-waveguide had been investigated by different research, since it permits to exploit inherent advantages of planar structures and NRD waveguide for low cost microwave application. However, several techniques were proposed, Grigoropoulos and Young present a non-radiative perforated dielectric (NRPD) in [6], Cassivi and $\mathrm{Wu}$ introduce the substrate integrated non-radiative dielectric (SINRD) [7]. Other scheme of design called engraved non-radiative dielectric waveguide (ENRD), proposed to reduce the problem of alignment and mechanical tolerances in fabrication of NRD components, this design was described in [8,9].

The reference [10] presents the design of pass band filter in hybrid architecture planar/non radiative dielectric wave guide integration technology and shows parameters S.

In order to optimize the results of this work, we thought to vary the width of the NRD guide and show the influence of this variation.

\section{Theoretical Approach}

Its basic component, the NRD waveguide consists of a rectangular-section dielectric rod sandwiched between conducting plates that are at a distance apart less than half the free-space wavelength, the thickness is $b$ and the relative dielectric constant is $\varepsilon_{r}$ which must be superior to the relative dielectric constant of the border dielectric.

To express the principle of operation mathematically, we will consider a straight strip as shown in Figure 1; the required distance between the two metallic plates of NRD wave guide is computed using the following relation:

$$
a<\frac{\lambda_{0}}{2 \sqrt{\varepsilon_{r}}}
$$

where $\lambda_{0}$ the free-space wavelength $\varepsilon_{r}$ the relative dielectric constant. 


\section{Results and Discussion}

The hybrid architecture planar/NRD waveguide integration geometry consist of a micro strip line deposited on the top of ground plane which is one of the two parallel metallic plates of the NRD wave guide. The coupling between the two dissimilar structures is achieved though apertures that are made in the common ground plane. The aperture orientation defines essentially the operating mode in the NRD wave guide.

The proposed structure of a design of pass band filter in hybrid architecture planar/NRD is presented in Figure 2. The filter is composed of a series of cylindrical dielectric resonators with a ray $b / 2$ and a height $a$, coupled by air gaps of length $d$.

The micro strip line is made of Rogers RT/Duroid 5880 dielectric substrate with $\varepsilon_{r}=2.3$ and thickness $h=$ $0.508 \mathrm{~mm}$ and the strip width of $w=1.55 \mathrm{~mm}$. The slot size have a length $l=6 \mathrm{~mm}$ and a width $w_{s}=1 \mathrm{~mm}$.

The characteristic of the cylindrical dielectrical NRD wave guide the ray its equal to $4.907 \mathrm{~mm}$ and the height $a=5.08 \mathrm{~mm}$, this cylindrical NRD waveguide is made of Rogers TMM-3 dielectric substrate $\varepsilon_{r}=3.27$. The parameters $l_{s}=1 \mathrm{~mm}$ and $l_{w}=4 \mathrm{~mm}$.

An absorbing boundary condition is applied in reducing our computional window for this unbounded structure so an electrical rectangular box is simulated (Figure $3)$.

Figure 4 shows the results for a variation of plus or minus $0.025 \mathrm{~mm}$ of the width of the waveguide. The

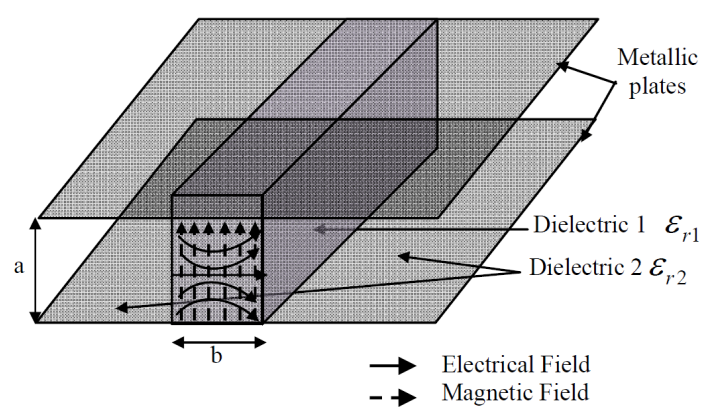

Figure 1. General structure of NRD waveguide and the distribution of the electric and magnetic fields.

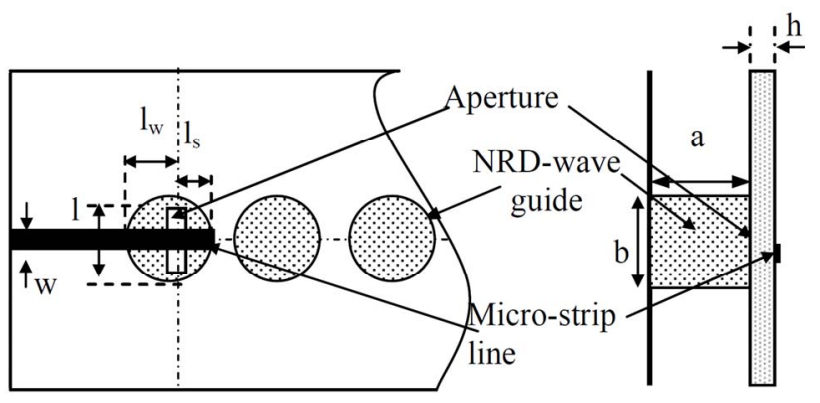

Figure 2. Structure design of cylindrical NRD waveguide.

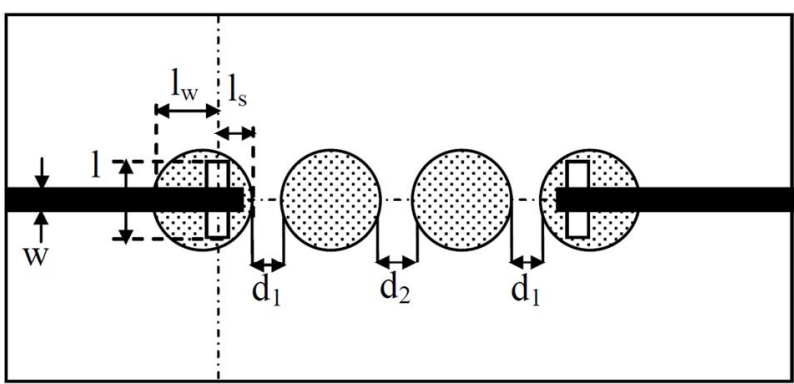

Figure 3. Dimension design for simulation.

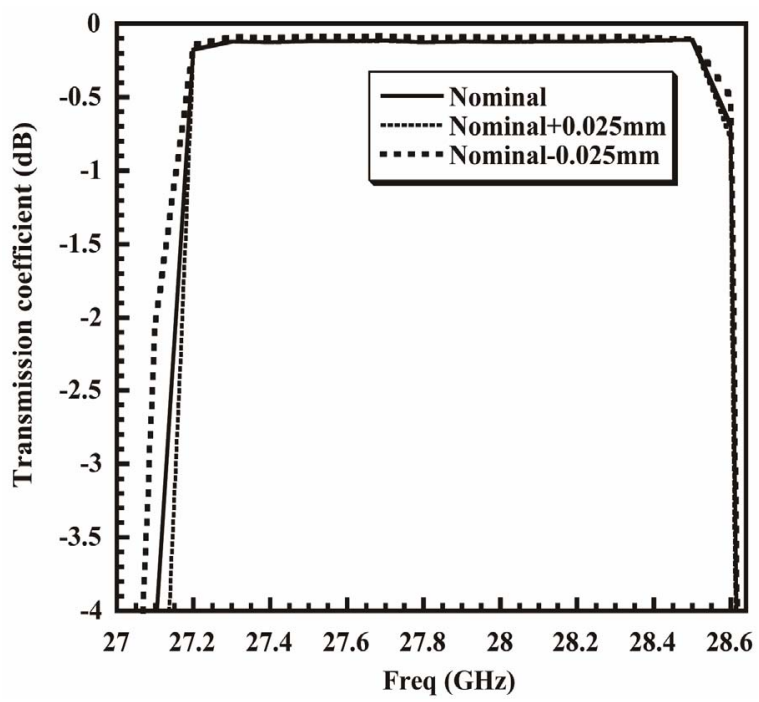

(a)

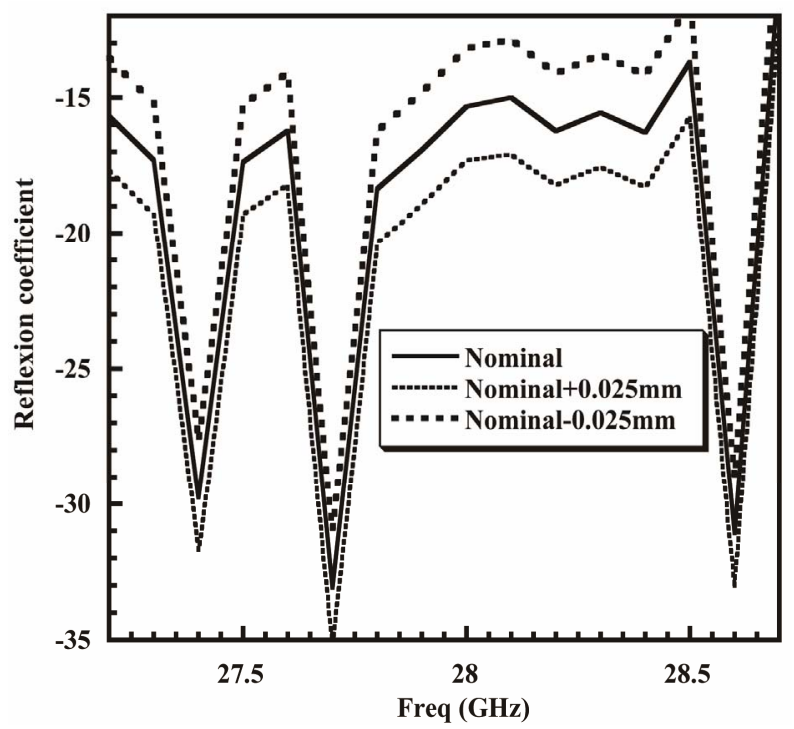

(b)

Figure 4. Sensitivity of width of the NRD waveguide: (a) Transmission coefficient; (b) Reflexion coefficient.

variation of the amplitude of the transmission and reflexion coefficients, but the adaptation of filter is not influenced by this variation of the width. 


\section{Conclusion}

We have presented the design of pass band filter in hybrid architecture planar/non radiative dielectric wave guide integration technology and the influence of the variation of the width of the NRD waveguide to the parameters $\mathrm{S}$.

\section{REFERENCES}

[1] T. Yoneyama and S. Nishida, "Nonradiative Dielectric Waveguide for Millimeter-Wave Integrated Circuits," IEEE Transactions on Microwave Theory and Technology, Vol. 29, No. 11, 1981, pp. 1188-1192. doi:10.1109/TMTT.1981.1130529

[2] T. Yoneyama, "Recent Development in NRD-Guide Technology,” Annales Des Télécommunications, Vol. 47, No. 11-12, 1992, pp. 508-514.

[3] T. Yoneyama and S. Nishida, "Non-Radiative Dielectric Waveguide,” In: K. J. Button, Ed., Infrared and Millimter Waves Series, 1984, pp. 61-98.

[4] A. Bacha and K. Wu "Toward an Optimum Design of NRD-Guide and Microstirp Line Transition for Hybrid Integration Technology," IEEE Transactions on MicroWave Theory and Technology, Vol. 46, No. 11, 1998, pp. 1796-1800.
[5] A. Bacha and K. Wu, "LSE-Mode Balun for Hybrid Integration of NRD-Guide and Microstrip Line," IEEE Micro-Wave and Guided Wave Letters, No. 5, 1998, pp. 199-201. doi:10.1109/75.668729

[6] N. Grigoropoulos and P. R. Young, "Low Cost Non-Radiative Perforated Dielectric Waveguide,” Europe MicroWave Conference, Munich, 7-9 October 2003, pp. 439442.

[7] Y. Cassivi and K. Wu, "Substrate Integrated Non-Radiative Dielectric Waveguide,” IEEE Micro-Wave Wireless Component Letters, Vol. 14, No. 3, 2004, pp. 89-91. doi:10.1109/LMWC.2004.824808

[8] Y. Cassivi, D. Deslandes and K. Wu, "Engraved NRDGuide for Millimeter-Wave Integrated Circuits,” IEEE MTT-S International Microwave Symposium Digest, Vol. 2, 2000, pp. 605-608.

[9] Y. Cassivi, D. Deslandes and K. Wu, "Design Considerations of Engraved NRD-Guide for Millimeter-Wave Integrated Circuits," IEEE Transactions on Microwave Theory and Technology, Vol. 50, No. 1, 2002, pp. 165-171. doi:10.1109/22.981261

[10] H. Harizi, L. Lattrach and A. Garsallah, "Design of Band Pass Filter in Hybrid Architecture Planar,” American Journal of Applied Science, Vol. 9, No. 10, 2012, pp. 1538-1541. 